\title{
Síndrome de hiperestimulación ovárica: (SHO) Revisión del tema
}

\author{
E. Otero; S. Bassil; S. Gordts; J. Donnez*
}

\begin{abstract}
Nuestro entendimiento de la fisiología de la ovulación continúa en progreso y desde la primera descripción de una inducción exitosa por Gemzell et al (1), muchos avances en el tratamiento han sido hechos. Estos tratamientos son ampliamente practicados en muchos países del mundo hoy en día. Varias sustancias farmacológicas como el citrato de clomifeno (CC), gonadotrofina menopáusica humana (HMG), hormona folículo estimulante purificada (FSH) y hormonas análogas de liberación gonadotrófica (GnRha) han sido usadas solas o en combinación con una u otra para inducir en grados diversos la estimulación ovárica. Las complicaciones médicas son un aspecto importante cuando se trabaja con inducción de la ovulación o en fertilización y transferencia de embriones (FIVET), transferencia intrafalopio de gametos (GIFT) y transferencia intrafalopio de zigotos (ZIFT). El síndrome de hiperestimulación ovárica (SHO) es la principal complicación asociada con estos tratamientos.
\end{abstract}

\section{Definición}

El SHO está representado por una gran variedad de manifestaciones clínicas y de laboratorio inducido por la administración exógena de sustancias usadas para estimular el crecimiento folicular y la ovulación. Está caracterizado por un agrandamiento ovárico bilateral, irritación peritoneal con ascitis y algunas veces por hemoconcentración, desequilibrio electrolítico, oliguria, derrame pleural y/o pericárdico e hipercoagulabilidad.

\section{Incidencia}

Una amplia variación ha sido reportada en la ocurrencia de varias categorías de SHO. La incidencia varía respectivamente entre 8 y $23 \%$ (2), 1 y $6 \%$ (3-5) y 0,1 y 4\% (3-9) para SHO leve, moderado y severo.

La alta incidencia ha sido asociada con tratamientos con gonadotrofinas (10-12) La inducción de la ovulación con CC y GnRha se ha visto menos relacionada al SHO y la forma severa es rara (13-17). El empleo de GnRha con una combinación de agentes estimulantes fue capaz de reducir la incidencia de SHO. Sin embargo el uso rutinario de GnRha en la mayoría de los programas de fertilización asistida no confirman esta teoría y por el contrario, su uso, paradójicamente incrementa la ocu-

Servicio de Ginecología. Universidad Católica de Lovaina. Clínica Universitaria. St-Luc, Bruxellas - Bélgica. rrencia $(7,18-19)$. El uso de FSH pura no altera la incidencia de SHO. El SHO 50\% (21-22), y su severidad ha sido previamente reportada que aumenta con la ocurrencia de embarazos múltiples $(11,23-24)$. Estos hallazgos no son confirmados por otros estudios.

\section{Clasificación}

Muchas clasificaciones han sido diseñadas con la evolución de las técnicas de reproducción asistida, la primera fue propuesta por Rabau et al (25), sin embargo, adaptaciones de esta clasificación fueron realizadas por la OMS en 1973 (26), por Schenker et al en 1978 (2) y por Golan et al en 1989 (27). Recientemente, Navot et al (28) propusieron adicionar una nueva categoría a la forma severa, que incluye las complicaciones que amenazan la vida en forma extrema "SHO crítico". Originalmente, el SHO presenta seis grados divididos en tres categorías clínicas, como se describen a continuación.

Grados 1 y 2 (Hiperestimulación Leve)

En el grado 1, existe una hiperestimulación bioquímica o niveles de estrógenos urinarios mayores de $150 \mathrm{~m} / 24$ horas o niveles de estradiol plasmático superiores a $200 \mathrm{pg} /$ $\mathrm{ml}$ y pregnanediol urinario superior a $10 \mathrm{mg} / 24$ horas. En el grado 2, existen síntomas clínicos tales como molestia abdominal, tensión y dolor y el agrandamiento ovárico no es superior a $5 \mathrm{~cm}$ de diámetro. Es difícil evaluar la incidencia de esta forma de $\mathrm{SHO}$ debido a la menor importancia de los hallazgos clínicos. Aunque la hiperestimulación leve está incluida en la clasificación del SHO, nosotros pensamos que esta forma tiene un valor clínico limitado y puede existir en algún grado en todas las pacientes tratadas con $\mathrm{HMG} /$ gonadotrofina coriónica humana (HCG) para inducción de la ovulación o para FIVET.

\section{Grados 3 y 4 (Hiperestimulación Moderada)}

En los grados 3 y 4 hay un incremento en la molestia abdominal acompañado por síntomas gastrointestinales tales como náusea, vómito y diarrea. Alguna ganancia de peso es observada, así como un marcado agrandamiento ovárico (hasta $12 \mathrm{~cm}$ ). El SHO moderado representa menos del $6 \%$ de los casos.

\section{Grados 5 y 6 (Hiperestimulación Severa)}

Los grados 5 y 6 son las formas severas del SHO. Ellos son caracterizados por la presencia de ascitis y algunas 
veces derrame pleural y pericárdico, desequilibrio electrolítico, hipovolemia y oliguria. Un marcado incremento en los síntomas clínicos, comparado con la forma moderada está presente. Disnea, hemoconcentración, incremento de la viscosidad sanguínea y fenómenos tromboembólicos pueden ser observados (2-13-19). El diámetro ovárico usualmente es superior a $12 \mathrm{~cm}$. Estos grados representan menos del $2 \%$ de todos los casos de SHO (2). En esta categoría Navot et al (28) han definido una entidad clínica adicional "SHO crítico". Esta clase incluye los aspectos clínicos más graves del SHO, tales como ascitis severa, síndrome de distress respiratorio agudo (SDRA), signos de falla renal, leucocitosis severa $(>25.000)$ y fenómenos tromboembólicos. Esta entidad ha sido recientemente reportada con una incidencia de $0,008 \%$ de todos los casos de SHO (19).

\section{Aspectos clínicos}

Los hallazgos clínicos del SHO aparecen de 3 a 10 días después de la ovulación o de la administración de HCG. Ellos son la manifestación de una combinación del agrandamiento ovárico y otras reacciones adversas, de acuerdo a la severidad de la presentación. Un marcado agravamiento puede ser observado si ocurre la implantación. En tales casos los síntomas pueden persistir hasta las 12 semanas de embarazo. En ausencia de concepción, una resolución relativamente rápida de los síntomas es observada (2 a 7 días). Recientemente, Dahl et al (5) distinguen dos entidades clínicas del SHO de acuerdo al tiempo de aparición de los síntomas. El SHO temprano y tardío ocurren respectivamente 3 a 10 días y 12 a 17 días posterior a la administración de la HCG. Aunque los síntomas clínicos son similares, los autores atribuyen un factor de riesgo diferente a cada entidad, con un papel preponderante del embarazo en la aparición del SHO tardío. En pacientes con SHO se debe tener cuidado en lo relacionado a embarazos múltiples y/o ectópicos, los cuales son relativamente frecuentes en ciclos en los que se produce la hiperestimulación. (11-21-29-30). Otras complicaciones ginecológicas deben ser tenidas en cuenta. Con el SHO leve, pueden estar presentes quistes lúteos múltiples bilaterales pudiendo llevar a una hemorragia intraperitoneal en caso de una ruptura y, excepcionalmente a una torsión anexial. Con el SHO moderado, los quistes ováricos son más grandes y pueden tomar hasta 30 días para resolverse (2-31). Con el SHO severo, una variedad de signos y síntomas complicados con hallazgos de laboratorio pueden estar presentes. En estos casos algunas muertes han sido reportadas (3-32).

\section{Complicaciones}

\section{Ascitis a tensión}

El grado de severidad del SHO podría determinarse de acuerdo con la extensión de la ascitis a tensión, la cual de acuerdo a su severidad puede llevar a hipovolemia u oliguria. Problemas de circulación sanguínea y respiratorios asociados a incrementos de la presión intraabdominal, se asocian con SHO severo. El derrame pleural puede también estar asociado con ascitis a tensión.

\section{Alteraciones Hematológicas}

Las alteraciones hematológicas de la hiperestimulación severa son caracterizadas por hipovolemia y hemoconcentración. Esas complicaciones son representadas por un incremento en el porcentaje del hematocrito y pueden estar asociadas con un aumento variable de la viscosidad sanguínea. Philips et al (35) han reportado algunas alteraciones en los parámetros de la coagulación, tales como aumento de los niveles del factor $\mathrm{V}$, plaquetas, fibrinógeno, protofibrinolisina y en la producción de tromboplastina. Sin embargo, Schenker y Weinstein (2) no confirmaron estos hallazgos en pacientes con hemoconcentración.

Algunos casos de trombosis venosa profunda y trombosis venosa braquial han sido descritos (19-36-38). La ocurrencia de fenómenos tromboembólicos (trombosis venosa profunda) en la ausencia de hemoconcentración y en la presencia de bajos niveles de antitrombina III también han sido documentados (39). Estos hallazgos apoyan la hipótesis del posible papel que juegan los altos niveles de estradiol $\left(E_{2}\right)$ y progesterona en la etiología de la trombosis venosa profunda asociada con el SHO. Otro reporte (40) describe un caso de accidente vascular cerebral isquémico asociado con una forma severa de SHO. Recientemente dos casos de trombosis venosa de la vena subclavia después de un SHO han sido reportados (41), resaltando la importancia del control hematológico en el seguimiento de este síndrome.

\section{Alteraciones Renales}

La insuficiencia renal aguda (IRA) es una de las más serias complicaciones del SHO severo. Está asociada con deficiente perfusión renal, probablemente relacionada a la hipocalcemia. Además factores tóxicos, tales como el uso de la indometacina como un inhibidor de la síntesis de las prostaglandinas en la prevención de la formación de un tercer espacio, podría también estar implicado en la patogénesis de la IRA (42-43). Las manifestaciones clínicas de la IRA son oliguria, desequilibrio electrolítico, azotemia e hiponatremia dilucional asociada con una hipersecreción de hormona antidiurética (ADH) (43). La corrección de la hipovolemia podría restaurar la perfusión renal y el flujo urinario. De hecho, un aumento en la producción urinaria indica el comienzo de la recuperación de un SHO.

\section{Alteraciones Hepáticas}

Signos de falla hepática han sido reportados durante las formas severas del SHO con y sin concepción (43-46). El daño hepatocelular y la colestasia han sido relacionados con niveles elevados de $\mathrm{E}_{2}$ y también como un signo de hepatotoxicidad probablemente inducido por la administración de indometacina (47).

\section{Alteraciones Pulmonares}

Las manifestaciones pulmonares de la forma severa del SHO incluyen derrame pleural asociado con vasoconstricción e incremento de la permeabilidad capilar, probablemente inducido por prostaglandinas. Estos hallazgos clínicos presentan en general un resultado relativamente benigno con 
manejo conservador. En algunos casos, sin embargo, síndrome de distress respiratorio agudo del adulto (SDRA) puede estar presente (19-48). La hipoxemia severa asociada con SDRA puede llevar a paro cardiorespiratorio, empeorando el pronóstico del SHO.

\section{Patogénesis}

Poco se conoce acerca de la patogénesis del SHO. Entre los mecanismos probables estaría un incremento en la permeabilidad capilar (2-49), probablemente relacionado con un aumento de la angiogénesis (50). Este fenómeno desempeña un papel importante en las manifestaciones clínicas: agrandamiento de los ovarios asociado con un incremento en la permeabilidad capilar ovárica, edema estromal intenso, ascitis y derrame pleural. Sin embargo, no se ha podido demostrar claramente que la ascitis se origine desde los ovarios (51). Principalmente dos sistemas se han visto comprometidos en el proceso, el sistema de las prostaglandinas y el de la reninaangiotensina (3). Esto da como resultado un paso rápido de líquido desde el espacio intravascular hacia un tercer espacio. Igualmente la hipovolemia, el aumento de la viscosidad y el tromboembolismo son consecuencia del aumento de la permeabilidad capilar con pérdida importante de líquido. El aumento de la reabsorción de agua y sal en el túbulo proximal renal, (52) así como la disminución en el intercambio de iones de hidrógeno y potasio en el túbulo distal producen oliguria, azotemia, hipercalcemia y acidosis metabólica.

El papel de las prostaglandinas (53) como la "sustancia activa" induciendo el fenómeno de retención de líquidos fue basado en la eficacia de los inhibidores de la síntesis de las prostaglandinas en la prevención de la ascitis, derrame pleural e hipovolemia. Esta teoría no fue confirmada por Balasch et al (54) quienes encontraron que la principal causa de la retención de líquidos es debida al incremento de la excreción urinaria de las prostaglandinas, antagonizando las alteraciones renales. Por otra parte, los estrógenos también han sido implicados en la patogenia del movimiento de fluidos. Soportando esta hipótesis, un incremento en la permeabilidad capilar ovárica y uterina han sido observadas en modelos de ratas (55). Sin embargo, este hallazgo no pudo ser confirmado con la administración de altas dosis de estrógenos a conejas (3). La extraperitonización de los ovarios y el hecho de que el SHO no puede reproducirse en machos, lleva a la suposición de que existe un mediador de origen ovárico, estimulado por los altos niveles estrogénicos directamente comprometido en los trastornos de la permeabilidad.

El sistema renina-angiotensina podría jugar un papel mayor en la angiogénesis y en el incremento de la permeabilidad capilar (56-57). Haning et al (58) y Montea et al (59) han encontrado niveles de renina plasmática elevada en pacientes con SHO asociado a un incremento de la aldosterona y la ADH. Sin embargo, ellos concluyen que estas modificaciones son secundarias a una excesiva secreción ovárica de una hormona desconocida que regula el fluido peritoneal.
La histamina también ha sido señalada como un posible mediador en la patogénesis del SHO (60-62) en modelos animales. Erlik et al (63) no pudieron demostrar ninguna diferencia en los niveles de histamina entre un grupo de conejas con SHO y un grupo control, concluyendo que la histamina en sí no desempeña ningún papel en la patogénesis de este síndrome. En conclusión, actualmente, la patogénesis de este síndrome puede ser explicada por un incremento súbito de la permeabilidad capilar mediado por una (hiper) secreción de una sustancia ovárica desconocida, que induce una activación de prostaglandinas, renina-angiotensina y sistema de la histamina, resultando en un paso de líquido desde el espacio intravascular a las cavidades peritoneal, pleural y pericárdica (tercer espacio). Los cambios hemodinámicos son debidos a una hipovolemia con descenso de la perfusión renal y de la excreción (oliguria), hemoconcentración, función hepática alterada, fenómenos tromboembólicos y con una última complicación, falla renal aguda con o sin SDRA.

\section{Tratamiento}

El tratamiento depende de la severidad del SHO. Las formas leves no requieren ninguna forma activa de terapia, tan sólo la observación (medidas de peso corporal, perímetro abdominal y volumen urinario diariamente). Las formas moderadas requieren una observación más estricta, debido a que éstas pueden pasar fácilmente a la forma severa, particularmente si ocurre un embarazo. Las formas severas requieren de hospitalización con una valoración diaria de electrólitos y del balance hídrico.

Basado en la teoría del papel de las prostaglandinas en el proceso de retención hídrica observado en las formas severas del SHO, Schenkeret et al (2) sugieren que la administración de un inhibidor de la síntesis de las prostaglandinas, tal como la indometacina podría tener un efecto beneficioso. Sin embargo estos resultados no son confirmados por otros, quienes fallaron en suprimir la ascitis en modelos animales tratados similarmente. Los riesgos teóricos de la indometacina en altas dosis contraindican el uso de estas sustancias durante el embarazo (65). También, Balasch et al (43) reportaron un caso de una paciente quien desarrolló una falla renal aguda con 2 días de administración de indometacina.

Otros agentes farmacológicos, tales como los inhibidores de la enzima convertidora de la angiotensina (66) y antihistamínicos (67) han sido propuestos en casos severos de SHO; pero no existen estudios controlados acerca de su eficacia y efecto embriogénico en mujeres embarazadas y ellos podrían ser usados solamente en situaciones en que su uso sea justificado.

El manejo ideal del SHO severo no ha sido completamente definido. En nuestra unidad, las pacientes son hospitalizadas, realizándose una evaluación diaria de peso y de la circunferencia abdominal, así como un balance hídrico estricto; una valoración de electrólitos plasmáticos y una ultrasonografía practicándose cada dos días. En ausencia de derrame pleural, desequilibrio electrolítico, oliguria y con un hematocrito $<45$, los líquidos son restringidos a $1000 \mathrm{ml} / 24 \mathrm{~h}$. Si dos reduccio- 
nes sucesivas en el tamaño ovárico son observadas, con una estabilización o disminución del peso y de la circunferencia abdominal, las pacientes son dadas de alta. Si por el contrario, alteraciones clínicas tales como desequilibrio electrolítico con hemoconcentración y oliguria están presentes, una perfusión con expansores plasmáticos y reposición de electrólitos es mandatoria. Nosotros usamos solución salina normal y albúmina humana para este propósito. En presencia de ascitis a tensión (> 2000 $\mathrm{ml}$ ) y signos de derrame pleural acompañado de disnea, una paracentesis abdominal (8-68) y excepcionalmente un drenaje pleural ofrecen resultados satisfactorios, especialmente en casos de SDRA (69). Los diuréticos están contraindicados por la reducción que existe en el compartimento intravascular, además se ha visto que son inefectivos para evacuar la acumulación de fluidos (263). Algunos autores prescriben diuréticos en asociación con una perfusión de albúmina, alcanzando resultados satisfactorios, pero agregan que esta terapia debe ser evitada en casos de hemoconcentración e hipotensión.

Un tratamiento profiláctico con anticoagulantes como heparina de bajo peso molecular, debe ser realizado en todos los casos de pacientes con SHO severo, aún en ausencia de transtornos de la coagulación o de fenómenos tromboembólicos.

Ferraretti et al (70) han administrado dopamina a pacientes con SHO severo para incrementar el flujo sanguíneo renal y la filtración glomerular. Estos autores concluyen que el tratamiento con dopamina es una herramienta prometedora en el manejo del SHO severo. Los procedimientos quirúrgicos deben ser evitados en casos de SHO. La sola indicación para cirugía es una sospecha de hemorragia intraperitoneal o una torsión anexial (71-72).

\section{Prevención de hiperestimulación ovárica}

La identificación de pacientes con riesgo de desarrollar SHO es el primer paso para la prevención. Para este propósito, varios criterios y medidas se han descrito.

\section{Estimulación Ovárica}

La estimulación de la ovulación en pacientes consideradas de riesgo (Síndrome de ovario poliquístico, o previos SHO) de desarrollar SHO debe ser extremadamente cuidadosa. El uso de bajas dosis de gonadotrofinas exógenas HMG o FSH o antiestrógenos (CC) por períodos un poco más prolongados es recomendado (73). Un incremento en las dosis de gonadotrofinas no es conveniente aún en presencia de niveles de $\mathrm{E}_{2}$ persistentemente bajos y con folículos pequeños. En estos casos es muy importante realizar un monitoreo estricto de la estimulación.

\section{Monitoreo de la Estimulación}

Las dos principales herramientas para el monitoreo de la ovulación son el $\mathrm{E}_{2}$ plasmático y la ecografía ovárica. Con base en la información obtenida, el médico puede diagnosticar el riesgo y tomar las medidas preventivas apropiadas. Es muy difícil establecer un límite de corte para los niveles de estradiol y los hallazgos ultrasonográficos. $\mathrm{El} \mathrm{SHO}$ ha sido observado con niveles de $\mathrm{E}_{2}$ de menos de $1000 \mathrm{pg} / \mathrm{ml}$ (3). Muy sensible en la identificación de las pacientes de riesgo es la inclinación en aumento del nivel del $\mathrm{E}_{2}$ plasmático (3-12). Valores de más del doble en 2 días, deben ser mirados como un serio signo de alarma. En relación a los test de $\mathrm{E}_{2}$, nosotros consideramos que cuando los niveles exceden de 2000 y $5000 \mathrm{pg} / \mathrm{ml}$, para la inducción de ovulación y ciclos de FIV, respectivamente, un cierto número de medidas preventivas deben ser aplicadas. El monitoreo ecográfico muestra un mejor valor predictivo que el $\mathrm{E}_{2}$ (10) en determinar las pacientes con riesgo de desarrollar el SHO. Sin embargo, no es suficiente para este propósito (12-20-74).

\section{Medidas Preventivas}

\section{Gonadotrofina coriónica humana ( $H C G$ )}

El SHO es reconocido como una patología esencialmente dependiente de HCG tanto endógena como exógena (2). La frecuencia de SHO fue inferior en pacientes que recibieron de 1000 a 5000 UI de HCG cuando se comparó con pacientes que recibieron de 5000 a 15000 UI (3). El uso cuidadoso de la HCG es el método más utilizado como medida preventiva del SHO.

\section{Aspiración folicular}

La aspiración de los folículos guiada por ultrasonografía, induce un descenso en los niveles hormonales, teniendo un efecto de protección en la ocurrencia del SHO (75-77). Sin embargo, este enfoque no ofrece una protección total y el SHO puede ocurrir (7-78). Por otro lado, la criopreservación de embriones obtenidos después de las aspiración selectiva de folículos ha permitido que las transferencias se realicen en ciclos posteriores, con lo cual se pueden evitar los posibles riesgos de un SHO severo (79).

Suspensión de la administración de gonadotrofina menopáusica humana (HMG)

Esta suspensión se ha visto como otra opción en la prevención del SHO. La utilidad de diferir 1 a 8 días la administración de HCG después de la última inyección de HMG ha sido recientemente confirmado por Urman et al (80). El principal objetivo es inducir un descenso en los niveles plasmáticos de $\mathrm{E}_{2}$ en pacientes de riesgo $\left(\mathrm{E}_{2}\right.$ $>5400 \mathrm{pg} / \mathrm{ml}$ ) y así permitir una completa maduración folicular. Este método ha permitido a los autores evitar la cancelación del ciclo, reduciendo drásticamente la incidencia de SHO y permitiéndoles mantener su promedio de embarazos. Sin embargo, Schenker et al (3) reportan un bajo promedio de embarazos con este procedimiento.

\section{Continuación de los análogos de GnRH.}

Salat-Baroux et al (81) y Forman et al (9) proponen descontinuar la administración de HMG, suspendiendo también la inyección de HCG y continuando con la administración de GnRHa como un método útil para prevenir el SHO. Estos autores han obtenido buenos resultados con este método en un grupo de pacientes de FIVET consideradas de riesgo de desarrollar una 
hiperestimulación. No obstante, Wada et al (82) no encontraron ningún efecto benéfico con el reposo ovárico cuando los análogos de GnRH fueron continuados.

Sustitución de la HCG por hormona luteinizante hipofisiaria humana purificada (LH) o análogos de GnRH

El uso de la HCG para producir la ovulación puede ser sustituido por la LH hipofisiaria humana purificada inyectada en forma repetida cada 24 horas, tratando así de imitar el pico normal de la LH (83). Itskovitz et al (84) y Empeire et al (85) han propuesto que en pacientes sometidas a estimulación ovárica sin análogos de $\mathrm{GnRH}$, se puede sustituir la HCG por GnRHa para la prevención del SHO con buenos resultados. El pick de LH hipofisiario inducido por GnRHa o LH hipofisiaria humana purificada, se ha visto que es más fisiológico que el pico inducido por la HCG, consecuente con esto se reduce el riesgo de hiperestimulación ovárica.

\section{Agentes farmacológicos}

La administración de glucocorticoides a pacientes de alto riesgo ha demostrado ser ineficaz en la prevención del SHO. Tan et al (86) no pudieron disminuir el SHO cuando usaron hidrocortisona y prednisolona durante 5 días, comenzando el día de la aspiración de los óvulos.

Recientemente Shohan et al (87) reportaron un efecto benéfico en la prevención del SHO severo cuando administraron albúmina humana en el momento de la aspiración folicular.

\section{Soporte de la fase lútea}

La administración de la HCG es conocida como un factor importante en el desarrollo del SHO; es lógico por esto que el uso de la HCG como soporte de la fase lútea haya sido asociado con una mayor incidencia del SHO (83). Este tipo de soporte debe ser evitado en los casos en los cuales los niveles de $\mathrm{E}_{2}$ plasmático estén muy elevados o exista un desarrollo folicular excesivo.

\section{Restricción de líquidos}

En nuestra unidad de fecundación in vitro, hemos realizado un estudio prospectivo randomizado para evaluar el efecto del reposo y de la restricción de líquidos en la aparición del SHO. En pacientes de riesgo $\left(\mathrm{E}_{2}>3500\right.$ $\mathrm{pg} / \mathrm{ml}$, número de folículos $>20$ ), estas medidas preventivas son iniciadas el día de la transferencia de embriones y son continuadas hasta la resolución del agrandamiento ovárico. Resultados preliminares (90) demuestran un significativo efecto de protección de estas medidas en la ocurrencia del SHO moderado y severo.

\section{Conclusiones}

A pesar de la mejoría en los protocolos usados para las inducciones de la ovulación, la patogénesis del SHO permanece desconocida. Aunque se han hecho progresos considerables en la prevención y tratamientos, todavía ocurren condiciones en las cuales se ve amenazada la vida de mujeres sanas tratadas solamente a causa de su infertilidad. Sin embargo, hasta el momento, existen dudas que el SHO pueda ser completamente evitado. Las estimulaciones ováricas y los tratamientos de inducción de la ovulación deben estar reservados a personas capaces de identificar los riesgos del SHO y que trabajen en unidades equipadas para un cuidadoso monitoreo de éstas y del manejo adecuado de todas las complicaciones.

\section{BIBLIOGRAFIA}

1. Gemzell CA., Diczfalusy E., Tillinger G. Clinical effect of human pituitary follicle stimulating hormone (FSH). J. Clin. Endocrinol Metab. 1958; 18: 1333.

2. Schenker JG and Weinstein D. Ovarian hyperstimulation syndrome: a current survey. Fertil Steril 1978; 30: 255-268.

3. Schenker JG. Prevention and treatment of ovarian hyperstimulation. Review. Hum. Reprod. 1993; 8: 653-659.

4. Bilan FIVNAT. Réponses aux stimulations de l'ovulation dans les procréations médicalement assistées (PMA). Contracept Fertil Sex 1987; 18: 592-594.

5. Dahl CA., Wheeler CA, Frishman GN., Hackett RJ., Seifer DB., Haning RV Jr. Early and late presentation of the ovarian hyperstimulation syndrome: two distinct entities with different risk factors. Hum Reprod 1994; 9: 792-799.

6. Asch RH., Li HP., Balmaceda JP., Weckstein LN., Stone SC. Severe ovarian hyperstimulation syndrome in assisted reproductive technology: definition of high risk groups. Hum. Reprod. 1991; 6: 1395-1399.

7. Golan A., Weinraub Z., Ron ER., Soffer Y., Herman A., Caspi E. Ovarian hyperstimulation syndrome following D-Trp -6 luteinizing hormone - releasing hormone microcapsules and menotropin for In Vitro Fertilization. Fertil Steril 1988; 50: 912-916.

8. Padilla S., Zamaris S., Bramki T., García J. Abdominal paracentesis for the ovarian hyperstimulation syndrome with severe pulmonary compromise. Fertil Steril 1990; 53: 365-367.

9. Forman R., Ross C., Frydman R., Barlow D., Egan D. Severe ovarian Hyperstimulation syndrome using agonists of gonadotropin-releasing hormone for In Vitro Fertilization: a European series and a proposal for prevention. Fertil Steril 1990; 53: 502-509.

10. Haning RV Jr., Austin CW., Carlson IH., Kuzma DL., ShapiroS., Zweibel WJ. Plasma estradiol is superior to ultrasound and urinary estriol glycuronide as a predictor of ovarian hyperstimulation during induction of ovulation with menotropins. Fertil Steril 1983; 40: 31-36.

11. Tulandi T., Meinnes RA., Arronet GH. Ovarian hyperstimulation syndrome following ovulation induction with hMG. Int. J. Fertil 1984; 29: 113-117.

12. Delvigne A., Dubois M., Battheu B., Bassil S., Meuleman C., De Sutter P., Rodesh C., Janssens P., Remacle P., Gordts S., Puttemans P., Joostens M., van Roosendael E., Leroy F. The ovarian hyperstimulation syndrome in In Vitro Fertilization: a Belgian multicentric study. II. Multiple discriminent analysis for rik prediction. Hum. Reprod. 1994; 8: 1361-1366.

13. Scommegna A., Lash SR. Ovarian overstimulation, massive ascites and singleton pregnancy after clomiphene. J. Am. Med. Assoc. 1969; 207: 753-758.

14. Southan AC., Janovsky NA. Massive ovarian hyperstimulation with clomiphene citrate. J. Am. Med. Assoc. 1962; 181: 443-447.

15. Morgan H., Paredes RA., Lachelin GCL. Severe ovarian hyperstimulation after clomiphene citarte in a hypothyroid patient. Case report Br. J. Obstet. Gynaecol. 1983; 90: 977-982.

16. Schriock ED., Jaffe RB. Induction of ovulation with gonadotrophinreleasing hormone Obstet. Gynaecol. Surv. 1986; 41: 414-423.

17. Gompel A., Mauvais-Jarvis P. Induction of ovulation with pulsatile GnRH in hypothalamic amenorrhea. Hum. Reprod. 1988; 3: 473-477. 
18. Groot CA., Lammers JW., Hollanders JM. Asthma after ovarian hyperstimulation syndrome. Lancet 1991; 12: 337.

19. Delvigne A., Demoulin A., Smitz J., Donnez J., Koninckx P., Dhont M., Englert Y., Delbeke L., Darcis L., Gordts S., Puttemans P., Gerris J., Schoysman R., Leroy F. The ovarian hyperstimulation syndrome in In Vitro Fertilization: a Belgian multicentric study. I. Clinical and biological features. Hum. Reprod. 1993; 8: 1353-1360.

20. Check JH., Wu CH., Gocial B., Adelson HG. Severe ovarian hyperstimulation syndrome from treatment with urinary follicle stimulating hormone: two cases. Fertil Steril 1985; 43: 317-319.

21. Tyler E. Treatment of anovulation with metropins. J. Am. Med. Assoc. 1969; 205: 16-22.

22. Schenker JG., Yarkoni S., Grant M. Multiple pregnancies following induction of ovulation. Fertil Steril 1981; 35: 105-123.

23. Caspi E., Ron-El R., Golam A., Nachum H., Herman A., Soffer Y., Weinraub Z. Results of in vitro fertilization and embryo-transfer by combined long-acting gonadotropin releasing hormone analog $\mathrm{D}$ Trp-6 luteinizing hormone-releasing hormone and gonadotropins. Fertil Steril 1989; 51: 95-99.

24. Mac Dougall MJ., Tan SL., Jacobs HS. In Vitro fertilization and the ovarian hyperstimulation syndrome. Hum. Reprod. 1992; 7: 597-600.

25. Rabau E., David A., Serr DM., Maschiach S., Lunenfeld B. Human menopausal gonadotropins for anovulation and sterility: results of 7 years of treatment. Am. J. Obstet. Gynaecol. 1967; 98: 92-98.

26. WHO Scientific Group. Agents stimulating gonadal functions in the human. Technical Report Series No. 154. Geneva: World Health Organization, 1973.

27. Golan A., Ron-El R., Herman A., Soffer Y., Weinraub Z., Caspi E. Ovarian hyperstimulation syndrome. An update review. Obstet. Gynaecol. Surv. 1989; 44: 430-440.

28. Navot D., Bergh PA., Laufer N. Ovarian hyperstimulation syndrome in novel reproductive technologies: prevention and treatment. Fertil Steril 1992; 58: 249-261.

29. Rizk B., Aboulghar MA., Mansour RT., Serour GI., Amin Y. Severe ovarian hyperstimulation syndrome, analytical study of twenty-one cases. Hum. Reprod. 6 (Suppl 1): 1991; 368-369.

30. Smitz J., Camus M., Devroey P., Erard P., Wisanto A., Van Steirteghem AC. Incidence of severe ovarian hyperstimulation syndrome after GnRH agonist/HM superovulation for in-vitro Fertilization. Hum. Reprod. 1990; 5: 933-937.

31. Schwartz M., Jewelewicz R. The use of gonadotropins for induction of ovulation. Fertil Steril 1981; 35: 3-12.

32. Mozes M., Bogowsky H., Anteby E., Lunenfeld B., Rabau E., Serr D., David A., Salmony M. Thromboembolic phenomena after ovarian stimulation with human menopausal gonadotrophins. Lancet ii, 1965; 1213-1215.

33. Glickman RM., Isselbacker KS. Abdominal swelling and ascites. In Petersdorf RL. and Adams RD (eds), Harrison principles of internal medicine. Mc Graw-Hill New York p. 209.

34. Robbins SL. Pathologic basis of diseases. Saunders Co. 9th edn. 1974; p. 1023.

35. Phillips LL., Gladstone W., van de Wiele R. Studies of the coagulation and fibrinolytic systems in hyperstimulation syndrome after administration of human gonadotropins. J. Reprod. Med. 1975; 14: 138-140.

36. Crooke AC., Butt WR., Palmer RF., Morris R., Edward RL., Anson CJ. Clinical trial of human gonadotropins. J. Obstet. Gynecol. Br. Common 1963; 70: 604-631.

37. Gemzell CA., Roos P., Loeffler E. Follicle stimulating hormone. In: SJ Behrman, RW Kistner (eds): Progress in infertility. Boston: Little, Brown and Co. 1975; 490.

38. Fournet N., Surrey E., Kerin J. Internal jugular vein thrombosis after ovulation induction with gonadotropins. Fertil Steril 1991; 56: 354356.

39. Kaaja R., Sieberg R., Koskimies A. Severe ovarian hyperstimulation syndrome and deep venous thrombosis. Lancet ii, 1989; 1043.

40. Rizk B., Meagher S., Fisher AM. Severe ovarian hyperstimulation syndrome and cerebrovascular accidents. Hum Reprod. 1990; 5: 697 . 698.

41. Mills MS., Eddowes HA., Fox R., Wardle PG. Subclavian vein thrombosis: a late complication of ovarian hyperstimulation syndrome Hum Reprod. 1992; 7: 370-371.
42. Demey HE., Delemans R., Galdermans D., Verpooten GA., De Broe ME., Bossaert LL. Acute oligo-anuria during ovarian hyperstimulation syndrome. Acta Obstet. Gynecol Scand 1987; 66: 741-743.

43. Balash J., Carmona F., Llach J., Arroyo V., Jové I., Vanrell J. Acute renal failure and liver dysfunction in a patient with severe ovarian hyperstimulation syndrome. Hum Reprod. 1990; 5: 348-351.

44. Ryley NG., Forman R., Barlow D., Fleming KA., Trowell JM. Liver abnormality in ovarian hyperstimulation syndrome. Hum. Reprod. 1990; 5: 938-943.

45. Sueldo CE., Price HM., Bachenberg K., Steinleitner A., Gitlin N., Swanson J. Liver dysfunction in ovarian hyperstimulation syndrome. A case report. J. Reprod. Med. 1988; 33: 387-390.

46. Younis JS., Zeevi D., Rabinowitz R., Laufer N., Schenker JG. Transient liver function tests abnormalities in ovarian hyperstimulation syndrome. Fertil Steril 1988; 50: 176-178.

47. Clive DM., Stoff JS. Renal syndromes associated with nonsteroidal anti-inflammatory drügs. N. Engl. J. Med. 1984; 310: 563-572.

48. Zosmer A., Katz Z., Lancet M., Konichezky S., Schwartz-Shoham Z. Adult respiratory distress syndrome complicating hyperstimulation syndrome ovarian. Fertil Steril 1987; 47: 524-527.

49. Tollan A., Holst N., Forsdahl F., Fadens HO., Oian P., Maltau JM. Transcapillary fluid dynamic during ovarian stimulation for in vitro fertilization. Am. J. Obstet. Gynecol. 1990; 162: 554-558.

50. Jacob W., Jentrsch KD, Maursberger B., Oehme P. Demonstration of angiogenesis activity in the corpus luteum of cattle. Exp Pathol 1977; 13: 231.

51. Yarali H., Fleige-Zahradka BG., Yuen BH., Mc Comb PF. The ascites in the ovarian hyperstimulation syndrome does not originate from the ovary. Fertil Steril. 1993; 59: 657-661.

52. Polishuk WK., Schenker JG. Ovarian overstimulation syndrome. Fertil Steril 1969; 20: 443-450.

53. Schenker JG., Polishuk WZ. An experimental model of ovarian hyperstimulation syndrome. In: Tischner M., Pilc J. (eds): Proceedings of the international Congress on Animal Reproduction. Vol.4. Krakow: Drukarnia Naukowa 1976; p. 635.

54. Balasch J., Arroyo V., Carmona F., Llach J., Jiménez W., Pare JC., Vanrell JA. Severe ovarian hyperstimulation syndrome: role of peripheral vasodilation. Fertil Steril 1991; 56: 1077-1083.

55. Davis JS. Hormonal control of plasma and erythrocyte volume of rat uterus. Am. J. Physiol 1960; 199: 841-846.

56. Navot D., Margalioth EJ., Laufer N., Birkenfeld A., Relon A., Rosler A., Schenker JG. Direct correlation between plasma renin activity and severity of ovarian hyperstimulation syndrome. Fertil Steril 1987; 48: 57-61.

57. Ong ACM., Eisen V., Rennie DP., Homburg R., Lachelin GCL. Jacobs HS., Slater JDH. The pathogenesis of the ovarian hyperstimulation syndrome (OHS): a possible role for ovarian renin. Clin Endocrinol 1991; 34: 43-49.

58. Haning RV Jr., Strawn EY., Nolten WE. Pathophysiology of the ovarian hyperstimulation syndrome. Obstet. Gynecol. 1985; 66: 220-224.

59. Montea E., Marana R., Garcea N., Caruso S. Iatrogenic hyperstimulation of the ovary with ascites: report of a case and considerations and management. Acta Eur Fertil 1977; 8: 155-157.

60. Knox GE., Dowd A., Spiesel S., Hong R. Antihistamine blockade of the ovarian hyperstimulation syndrome. II. Possible role of antigenantibody complexes in the pathogenesis of the syndrome. Fertil Steril 1975; 26: 418-421.

61. Gergly RZ., Paldi E., Erlik Y., Makler A. Treatment of ovarian hyperstimulation syndrome by antihistamine. Obstet. Gynecol. 1976; 47: 83-85.

62. Pride SM., Ho Yuen B., Moon YS. Clinical endocrinological and intra-ovarian prostaglandin- $\mathrm{F}$ response to $\mathrm{H}-1$ receptor blockade in the ovarian hyperstimulation syndrome. Am. J. Obstet. Gynecol. 1984; $148: 670-674$

63. Erlik Y., Naot Y., Friedman M., Ben-David E., Paldi E. Histamine levels in ovarian hyperstimulation syndrome. Obstet. Gynecol. 1979; 53: $580-582$.

64. Borenstein R., Elhalah U., Luchelfed B., Schwartz ZS. Severe ovarian hyperstimulation syndrome: a reevaluated therapeutic approach. Fertil Steril 1989; 51: 791-795.

65. Katz Z.,Lancet M., Borenstein R., Chemke J. Absence of teratogenicity of indomethacin in ovarian hyperstimulation syndrome. Int. J. Fertil. 1984; 29: 186-188. 
66. Schenker JG., Navot D. Complication of induction of ovulation. In Teoh ES:, Ratnam SS and Wong C. (eds): Ovulation and Early Pregnancy. The Parthenon Publishing Group, Carnforth, Lacashire 1987; p. 53.

67. Kirshon B., Doody M., Cotton D., Gibbons W. Management of ovarian hyperstimulation syndrome with chlorpheniramine maleate, mannitol and invasive hemodynamic monitoring. Obstet. Gynecol. 1988; 71: 485-487.

68. Aboulghar MA., Mansour RT., Serour GI., Amin Y. Ultrsonically guided vaginal aspiration of ascites in the treatment of severe ovarian hyperstimulation syndrome. Fertil Steril 1990; 53: 933-935.

69. García-Compean D., Pérez-Martínez L., Guerra JM., Barragán RF. Therapeutic paracentesis with albumin infusion for the treatment of tension ascites in cirrhotic patients. Rev. Gastroenterol. Mex. 1990; 55: 7-12.

70. Ferraretti AP., Gianaroli L., Diotallevi L., Festi C., Trounson A. Dopamine treatment for severe ovarian hyperstimulation syndrome. Hum. Reprod. 1992; 7: 180-183.

71. Kemmann E., Ghazi DM., Corson GH. Adnexal torsion in menotropin induced pregnancies. Obstet. Gynecol. 1990; 3: 403-406

72. Mashiach S., Bider D., Moran O., Goldberg M., Ben-Rafael Z. Adnexal torsion of hypestimulated ovaries in pregnancies after gonadotropin therapy. Fertil Steril 1990; 53: 76-80.

73. Franks S., Mason HD., Polson DW., Winston RML., Margara R., Reed MJ. Mechanism and management of ovulatory failure in women with polycistic ovary syndrome. Hum. Reprod. 1988; 3: 531-544.

74. Mc Ardle C., Seibel M., Hann L., Weinstein F., Taymor M. The diagnosis of ovarian hyperstimulation (OHS): the impact of ultrasound. Fertil Steril 1983; 39: 464-467.

75. Belaisch-Allart J., Testart J., Belasich J., Frydman R., Hazout A. Selective ovocyte retrieval: a new approach to ovarian hyperstimulation. Fertil Steril 1988; 50: 654-656.

76. Ingerslev HJ. Selective follicular reduction following by exogenous gonadotrophins in polycystic ovarian disease. A new approach to treatment. Hum. Reprod. 1991; 6: 682-685.

77. Rabinowitz R., Laufer N., Lewin A., Schenker JG. Rate of ovarian hyperstimulation syndrome after high-dose HMG for induction of ovulation in IVF cycles. 5th Congress on IVF-ET. Norfolk, USA. Abstract 500 1987; 111.

78. Friedman CI., Schmidt GE., Chang FE., Kim MH. Severe ovarian hyperstimulation following follicular aspiration. Am. J. Obstet. Gynecol. 1984; 150: 436-437.

79. Wada I., Matson PL., Troup SA., Moroll D., Hunt L., Lieberman BA. Does elective cryopreservation of all embryos from women at risk of ovarian hyperstimuiation syndrome reduce the incidence of the condition. Br. J. Obstet. Gynecol. 1993; 100: 265-269.

80. Urman B., Pride SM., Yuen BH. Management of overstimulated gonadotrophin cycle with a controlled drift period. Hum. Reprod. 1992; 7: 213-217.

81. Salat-Baroux F., Alvarez S., Antoine JM., Cornet D., Tibi C., Plachot M., Mandelbaum J. Treatment of hyperstimulation during in vitro fertilization. Hum. Reprod. 1990; 5: 36-39.

82. Wada I., Matson PL., Horne G., BuckP.,Lieberman BA. Is continuation of a gonadotrophin-releasing hormone agonist ( $\mathrm{GnRHa}$ ) necessary for women at risk of developing the ovarian hypeerstimulation syndrome? Hum. Reprod. 1992; 7: 1090-1093.

83. Crosignani PG., Trojsi L., Attanasio A., Tonani E., Donini P. Hormonal profiles in anovulatory patients treated with gonadotropins and synthetic luteinizing hormone releasing hormone. Obstet. Gynecol. 1975; 46: 15-22.

84. Itskowitz J., Boldes R., Levron J., Erlik Y., Kabana L., Brandes JM. Induction of preovulatory luteinizing hormone surge and prevention of ovarian hyperstimulation syndrome by gonadotropin-releasing hormone agonist. Fertil Steril 1991; 56: 213-220.

85. Emperaire JC., Ruffie A. Triggering ovulation with endogenous luteinizing hormone may prevent the ovarian hyperstimulation syndrome. Hum. Reprod. 1991; 6: 506-510.

86. Tan SL., Balen A., El Hussein E., Campbell S., Jacobs HS. The administration of glucocorticoids for prevention of ovarian hyperstimulation syndrome in In vitro fertilization: a prospective randomized study. Fertil Steril 1992; 58: 378-383.

87. Shoham Z., Weissman A., Barash A., Borenstein R., Schachter M., Insler $\mathrm{V}$. Intravenous albumin for the prevention of severe ovarian hyperstimulation syndrome in In vitro fertilization program: a prospective, randomized, placebo-controlled study. Fertil Steril 1994; 62: 137-142.

88. Herman A., Ron-El R., Golan A., Raziel A., Soffer Y., Caspi E. Pregnancy rate and ovarian hyperstimulation after luteal human chronic gonadotropin in In vitro fertilization stimulated with gonadotropin-releasing hormone analog and menotropins. Fertil Steril 1990; 52: 92-96.

89. Mc Clure N., Leya J., Radwanska E., Rawlins R., Haning RV jr. Luteal phase support and severe ovarian hyperstimulation syndrome. Hum. Reprod. 1992; 7: 758-764.

90. Bassil S., Godin PA., Stallaert S., Nisolle M., Donnez J., Gordts S. Ovarian hyperstimulation syndrome: do preventive measures work? Hum. Reprod. 9 (suppl 4). abs. 412. 1994; 156. 